\title{
Robust Eigenvalue Assignment via Sampled State-Feedback for Linear Polytopic Discrete-Time Periodic Systems
}

\author{
Pedro H. S. Coutinho* Thiago P. das Chagas ${ }^{* *}$ \\ Leonardo A. B. Torres ${ }^{* * *}$ Reinaldo M. Palhares ${ }^{* * *}$ \\ * Graduate Program in Electrical Engineering, Federal University of \\ Minas Gerais, MG (e-mail: phcoutinho@ufmg.br). \\ ** Mechatronics Laboratory, Universidade Estadual de Santa Cruz, BA \\ (e-mail: tpchagas@uesc.br) \\ *** Department of Electronics Engineering, Federal University of Minas \\ Gerais, MG (e-mails:leotorres@ufmg.br,rpalhares@ufmg.br)
}

\begin{abstract}
This work deals with the robust eigenvalue assignment problem for periodic discretetime systems with polytopic uncertainties. By employing a sampled state-feedback control law, a suitable time-invariant reformulation describing a period ahead dynamics associated with the uncertain periodic system is obtained. The obtained results are based on a special class of parameter-dependent Lyapunov functions that adequately cope with the one period ahead dynamics. The robust eigenvalue assignment problem is solved via a convex problem. The proposed conditions ensure that all eigenvalues of the proposed time-invariant reformulation subject to uncertainties are within a specified circular region in the complex plane. Numerical simulations are presented to illustrate the effectiveness of the proposed conditions.
\end{abstract}

Keywords: Robust control; Periodic systems; Sampled state-feedback control; Polytopic uncertainty; Linear matrix inequality.

\section{INTRODUCTION}

In modern control theory, pole (or eigenvalue) assignment via state-feedback has become a popular strategy for modifying the closed-loop dynamic response of linear systems. A number of processes present in a variety of areas can be modeled as periodic linear systems. Therefore, they constitute an important subclass of general linear systems (Bittanti and Colaneri, 2009; Sreedhar and Van Dooren, 1993). Well-known applications include satellite attitude control and vibration attenuation in helicopters (Bittanti and Cuzzola, 2002), stabilization of unstable periodic orbits in chaotic systems (Chagas et al., 2018), wind turbine control and multirate sampled data systems and others (see Yang (2018) and references therein).

One of the widespread approaches for analysis and synthesis of linear periodic systems is based on their conversion to some equivalent linear time-invariant representation (TIR). It allows employing well-established tools developed for linear time-invariant (LTI) systems (Lv et al., 2010). A common procedure for that is the discrete-time lifting proposed by Meyer and Burrus (1975). Based on this TIR for periodic discrete-time systems, important results have been reported such as periodic Lyapunov and Riccati equations (Bittanti and Colaneri, 2009), pole placement (Colaneri, 1991; Sreedhar and Van Dooren, 1993) and Linear Quadratic optimal control.

\footnotetext{
‡ This work was supported in part by the Brazilian agencies CAPES, CNPq, and FAPEMIG.
}

In practical applications, systems are usually affected by parametric uncertainties. Taking them into account in the control design is crucial to ensure closed-loop performance and stability. Based on the Lyapunov stability theory, several approaches have been proposed for robust control of periodic systems with polytopic uncertainties. One of the greatest advantages in considering the polytopic description is that synthesis conditions can be derived in the form of linear matrix inequalities (LMIs), which can be efficiently solved using semidefinite programming (Löfberg, 2004). Examples of robust control techniques for this class of periodic systems include state and output-feedback design (De Souza and Trofino, 2000), state-feedback control with $\mathscr{H}_{2}$ performance (Farges et al., 2007) and periodically time-varying memory state-feedback controller (Ebihara et al., 2011), which was extended for $\mathscr{H}_{\infty}$ synthesis (Trégouët et al., 2012). Recently, robust stability analysis conditions have been proposed for periodic systems with timevarying uncertainties (Agulhari and Lacerda, 2018) and observer-based control design for discrete-time uncertain periodic systems has been tackled by Agulhari and Lacerda (2019) and Keles et al. (2019).

With respect to eigenvalue assignment for uncertain periodic systems, previous results (Colaneri, 1991; Sreedhar and Van Dooren, 1993) can not be directly applied because they do not guarantee the closed-loop stability/performance in the presence of uncertainties. Based on solutions of generalized Sylvester matrix equations, robust eigenvalue assignment was solved in Lv et al. (2010) by converting it into a nonconvex optimization problem. 
Motivated by the aforementioned discussion, this work tackles the problem of eigenvalue assignment for the class of linear discrete-time periodic systems subject to polytopic uncertainties. By employing a sampled periodic state-feedback control scheme, a TIR (also affected by uncertainties) is obtained. Since precise eigenvalue assignment is not possible due to the presence of uncertainties, the $\mathscr{D}$-stability concept for uncertain LTI systems (Chilali and Gahinet, 1996; Mao and Chu, 2009) is exploited to assign the closed-loop eigenvalues in a specified region in the complex plane. In addition, as the TIR corresponds to one period ahead dynamics, the application of conventional Lyapunov functions is not direct. Thus, the proposed conditions are based on a special class of Lyapunov functions employed by Kruszewski et al. (2008) in the context of " $k$-samples variation" approach for fuzzy systems. In contrast to Kruszewski et al. (2008), in which the step ahead variation " $k$ " is arbitrarily selected, here, it is appropriately defined as the system period to incorporate the one period ahead dynamics. Finally, a systematic procedure is provided to obtain tractable LMI design conditions.

The structure of this paper is as follows. In Section 2, it is presented the discrete-time linear periodic system with polytopic uncertainties and the related TIR. The main results of this work are in Section 3, a sufficient condition for robust eigenvalue assignment and the procedure to obtain LMI design conditions. The effectiveness of the proposed approach is illustrated in Section 4. Finally, conclusion is given in Section 5 .

Notation The space of real symmetric matrices of order $n$ is denoted by $\mathbb{S}^{n}$. The set $I_{[1, p]} \subset \mathbb{N}$ denotes $\{1, \ldots, p\}$. The identity matrix of order $n$ is denoted by $I_{n}$ and null matrix of appropriate dimension by $0 . \Lambda(X)$ denotes the spectrum of $X$. In the product of sequences of matrices, right multiplication is considered, i.e., $\prod_{i=1}^{N} X_{i}=X_{1} X_{2} \cdots X_{N}$.

\section{PROBLEM FORMULATION}

Consider the linear $p$-periodic discrete-time system subject to a polytopic uncertainty described by the difference equation:

$$
x_{k+1}=A_{k}(\theta) x_{k}+B_{k}(\theta) u_{k},
$$

where $k \in \mathbb{N}$ is the time index, $x_{k} \in \mathbb{R}^{n}$ is the state vector, $u_{k} \in \mathbb{R}^{m}$ is the input vector, $A_{k}(\theta) \in \mathbb{R}^{n \times n}$ is the state matrix and $B_{k}(\theta) \in \mathbb{R}^{n \times m}$ is the input matrix. The periodicity of the system is verified by

$$
A_{k+p}(\theta)=A_{k}(\theta), B_{k+p}(\theta)=B_{k}(\theta), p \in \mathbb{N}^{*} .
$$

In addition, the system matrices belong to a polytopic domain parameterized by the time-invariant parameter $\theta$, such that

$$
\left(A_{k}(\theta) B_{k}(\theta)\right)=\sum_{i=1}^{N} \theta_{i}\left(A_{k}^{[i]} B_{k}^{[i]}\right),
$$

where $N$ is the number of polytope's vertices, and $\theta$ belongs to the following standard unit simplex:

$$
\Theta_{N}=\left\{\theta \in \mathbb{R}^{N}: \sum_{i=1}^{N} \theta_{i}=1, \theta_{i} \geq 0, i \in I_{[1, N]}\right\}
$$

Observe that each polytope vertex $\left(A_{k}^{[i]}, B_{k}^{[i]}\right)$ is $p$-periodic, which implies that the polytopic domain varies periodically as well (Agulhari and Lacerda, 2018).

Hereafter, we denote by $\Phi\left(\tau_{2}, \tau_{1}, \theta\right)$ the state transition matrix of (1) with $u_{k} \equiv 0$ :

$$
\Phi\left(\tau_{2}, \tau_{1}, \theta\right) \triangleq \begin{cases}I_{n}, & \tau_{2}=\tau_{1} \\ \prod_{m=1}^{\tau_{2}-\tau_{1}} A_{\tau_{2}-m}(\theta), & \tau_{2}>\tau_{1} .\end{cases}
$$

Due to periodicity, the following property is verified: $\Phi\left(\tau_{2}+p, \tau_{1}+p, \theta\right)=\Phi\left(\tau_{2}, \tau_{1}, \theta\right)$. In addition, the monodromy matrix at $\tau$ is defined by:

$$
\begin{aligned}
\Psi(\tau, \theta) & \triangleq \Phi(\tau+p, \tau, \theta) \\
& =\prod_{m=1}^{p}\left(\sum_{i_{p-m}=1}^{N} \theta_{i_{p-m}} A_{\tau+p-m}^{\left[i_{p-m}\right]}\right) \\
& =\sum_{i_{p-1}=1}^{N} \ldots \sum_{i_{0}=1}^{N} \prod_{m=1}^{p} \theta_{i_{p-m}} A_{\tau+p-m}^{\left[i_{p-m}\right]}
\end{aligned}
$$

The eigenvalues of $\Psi(\tau, \theta)$ are called characteristic multipliers. For a shorthand notation, we define the multisimplex variable $\zeta \in \Theta_{N}^{p}$ such that the monodromy matrix can be rewritten as follows:

$$
\Psi(\tau, \zeta)=\mathcal{A}(\zeta)=\sum_{\mathbf{i} \in \mathbb{I}_{p}} \zeta_{\mathbf{i}} \mathcal{A}^{[\mathbf{i}]}
$$

where $\mathcal{A}^{[\mathbf{i}]}=\prod_{m=1}^{p} A_{\tau+p-m}^{\left[i_{p-m}\right]}$, and $\mathbf{i}=\left(i_{p-1}, \ldots, i_{0}\right)$ is a multi-index defined within the index set:

$$
\mathbb{I}_{p} \triangleq\left\{\mathbf{i}=\left(i_{p-1}, \ldots, i_{0}\right): i_{j} \in I_{[1, N]}, j \in I_{[0, p-1]}\right\}
$$

Notice that $\mathcal{A}(\zeta)$ is a homogeneous polynomial matrix of order $p$ with respect to the parameter $\theta$.

\subsection{Deriving the Time-Invariant Reformulation}

The considered control scheme, called sampled feedback control (SFC), is based on the sample and hold strategy (Bittanti and Colaneri, 2009). It consists in applying the input signal using one sampled state per cycle, as defined in the following control law:

$$
u_{k}\left(x_{l}\right)=K_{k} x_{l}, \quad k \in I_{[l, l+p-1]},
$$

where $l=i p+\tau, i \in \mathbb{N}, K_{k} \in \mathbb{R}^{m \times n}$ is a $p$-periodic gain matrix to be designed and $\tau \in \mathbb{N}$ is a fixed tag in time related to the initial condition.

The sampling scheme is illustrated in Figure 1 for a system with period $p=3$ and $\tau=1$. The time instants when the system state is sampled and the start of the sequence of $p$ control actions is applied are marked with ' $\bullet$ ' in the discrete-time axis ' $k$ '.

After substituting (5) into (1), the closed-loop dynamics for one period $p$ ahead becomes:

$$
x_{l+p}=(\mathcal{A}(\zeta)+\mathcal{B}(\zeta) \mathcal{K}) x_{l},
$$

where $\mathcal{A}(\zeta)$ is defined in $(4), \mathcal{B}(\zeta) \triangleq\left(\mathcal{B}_{1} \cdots \mathcal{B}_{p}\right)$, with

$$
\begin{aligned}
& \mathcal{B}_{j}=\Phi(\tau+p, \tau+j, \theta) B_{\tau+j-1}(\theta) \\
& =\sum_{i_{p-1}=1}^{N} \ldots \sum_{i_{j-1}=1}^{N} \prod_{m=j}^{p} \theta_{i_{p-m}} A_{\tau+p-m}^{\left[i_{p-m}\right]} B_{\tau+j-1}^{\left[i_{j-1}\right]}
\end{aligned}
$$




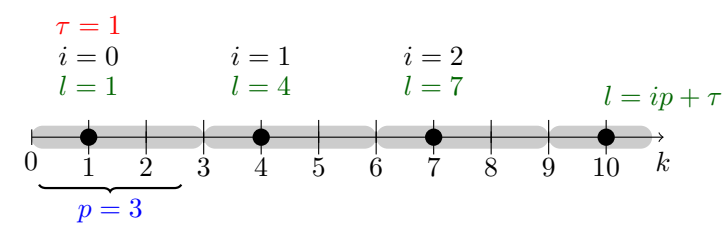

Figure 1. Sampling strategy of SFC for $p=3$ and $\tau=1$. The sampling times are marked with ' $\bullet$ ', but control actions are applied every time step using the last sampled information as detailed in (5).

for $j \in I_{[1, p]}$, and $\mathcal{K} \triangleq\left(K_{\tau}^{\top} \cdots K_{\tau+p-1}^{\top}\right)^{\top}$. The system (6) will be referred as the time-invariant reformulation of (1) in feedback with (5). As expected, the TIR is also affected by the uncertain parameters.

As pointed out by Colaneri (1991), in adopting the SFC scheme in (5), the system dynamics is affected by latent variables due to the sample and hold device governed by the following state periodic equations:

$$
\begin{aligned}
v_{k+1} & =S_{k} v_{k}+Q_{k} x_{k} \\
u_{k} & =K_{k} S_{k} v_{k}+K_{k} Q_{k} x_{k},
\end{aligned}
$$

where $Q_{k} \triangleq I_{n}-S_{k}$, and $S_{\tau}=0_{n}, S_{k}=I_{n}$, for all $k \in I_{[\tau+1, \tau+p-1]}$. Thus, it is possible to write the dynamics of the $2 n$-dimensional extended state vector $\left(x_{k}^{\top} v_{k}^{\top}\right)^{\top}$ as follows:

$$
\begin{aligned}
& \left(\begin{array}{l}
x_{k+1} \\
v_{k+1}
\end{array}\right)=\widetilde{A}_{k}(\theta)\left(\begin{array}{l}
x_{k} \\
v_{k}
\end{array}\right), \\
& \widetilde{A}_{k}(\theta)=\left(\begin{array}{cc}
A_{k}(\theta)+B_{k}(\theta) K_{k} Q_{k} & B_{k} K_{k} S_{k} \\
Q_{k} & S_{k}
\end{array}\right) .
\end{aligned}
$$

By computing the monodromy matrix of the last system dynamics at $\tau$, one has:

$$
\left(\begin{array}{cc}
\mathcal{A}(\zeta)+\mathcal{B}(\zeta) \mathcal{K} & 0 \\
I_{n} & 0
\end{array}\right)
$$

Then, it is clear that the $n$ nonzero eigenvalues of the above matrix are exactly those of (6). In addition, the eigenvalues of $\mathcal{A}(\zeta)$ coincide with the characteristic multipliers of the monodromy matrix $\Psi(\tau, \theta)$ of the unforced (or input-free) system (1).

From the Floquet theory, the unforced system (1) is asymptotically stable if and only if all the characteristic multipliers belong to the open unit disk in the complex plane (Bittanti and Colaneri, 2009). Thus, the stability of (1) in feedback with the control law (5) can be studied using the TIR in (6) with the advantage of employing well established results in the context of uncertain LTI systems such as $\mathscr{D}$-stability.

This analysis follows for other structural properties. In particular, a useful result is that system (6) is controllable if and only if system (1) is controllable (Bittanti and Colaneri, 2009). Thus, it is possible to design the periodic control gain $K_{k}, k \in I_{[l, l+p-1]}$, based on the uncertain TIR.

\subsection{Eigenvalue Assignment via $S F C$}

For motivation, consider a $p$-periodic discrete-time linear system without uncertainties

$$
x_{k+1}=A_{k} x_{k}+B_{k} u_{k}
$$

in feedback with control law (5), which produces the following closed-loop dynamics

$$
x_{l+p}=(\mathcal{A}+\mathcal{B K}) x_{l},
$$

with $\mathcal{A}$ and $\mathcal{B}$ obtained as previously but not considering uncertainties. In this case, the problem of eigenvalue assignment via $\mathrm{SFC}$ is stated as follows.

Problem 1. Design a matrix $\mathcal{K}$ such that $\Lambda(\mathcal{A}+\mathcal{B K})=\Gamma$, where $\Gamma \in \mathbb{C}^{n}$ is a vector of $n$ specified eigenvalues.

It is clear that the eigenvalue assignment, as stated in Problem 1, for periodic uncertain systems as (1) is much more involved since the eigenvalues of $(\mathcal{A}(\zeta)+\mathcal{B}(\zeta) \mathcal{K})$ are affected by the polytopic uncertainties. Thus, designing a matrix $\mathcal{K}$ to ensure $\Lambda(\mathcal{A}(\zeta)+\mathcal{B}(\zeta) \mathcal{K})=\Gamma$, or at least in its vicinity, may not be possible.

Alternatively, the idea to be exploited here is suitably selecting a region in the complex plane, instead of a unique point $\Gamma \in \mathbb{C}^{n}$, for which all the eigenvalues of (6) should be assigned for all admissible polytopic uncertainties.

The region considered for the eigenvalue assignment problem is

$$
\mathscr{D}(\alpha, r) \triangleq\{z \in \mathbb{C}:|z-\alpha|<r\},|\alpha|+r<1,
$$

which represents a disk in the complex plane with radius $r$ and centered at $\alpha+j 0$. This is a typical region for discretetime systems since it is possible to allocate the eigenvalues such that usual closed-loop performance specifications such as decay rate and damping ratio can be ensured (Chilali and Gahinet, 1996; Mao and Chu, 2009). Based on that, the control problem to be addressed here is stated as follows.

Problem 2. Design a matrix $\mathcal{K}$ such that all eigenvalues of (6) belong to the region $\mathscr{D}(\alpha, r)$ in the complex plane.

If the above control problem is solved, the closed-loop system (6) is said to be $\mathscr{D}(\alpha, r)$-stable.

\section{MAIN RESULT}

In this section, a sufficient condition to solve Problem 2 is presented. As this condition is given in terms of the multisimplex variable $\zeta$, a procedure to derive a finite set of LMI conditions to solve it is presented.

The conditions are derived regarding Lyapunov stability theory. However, as the time-invariant reformulation (6) is written as a one period $p$ ahead dynamics, usual Lyapunov functions such as quadratic $V\left(x_{l}\right)=x_{l}^{\top} P^{-1} x_{l}$ or even parameter-dependent as $V\left(x_{l}\right)=x_{l}^{\top} P(\zeta)^{-1} x_{l}$ can not be directly employed because the $p$ ahead dynamics can not be properly introduced in the one step ahead variation $V\left(x_{l+1}\right)-V\left(x_{l}\right)$. As an alternative, the proposed conditions are based on the following Lyapunov function candidate:

$$
V\left(x_{l}\right)=\sum_{i=1}^{p} x_{l+i-1}^{\top} P(\zeta)^{-1} x_{l+i-1}
$$

where $P(\zeta) \succ 0 \in \mathbb{S}^{n}$ and $p \in \mathbb{N}^{*}$ is the period of system (1). This Lyapunov function candidate is based on the one used by Kruszewski et al. (2008). By computing the difference $\Delta V=V\left(x_{l+1}\right)-V\left(x_{l}\right)$, a condition to ensure the origin of (6) is asymptotically stable, i.e., all their 
eigenvalues belong to the open unit disk in the complex plane, is given by the following inequality:

$$
\Delta V=x_{l+p}^{\top} P(\zeta)^{-1} x_{l+p}-x_{l}^{\top} P(\zeta)^{-1} x_{l}<0,
$$

which is a difference between the state function evaluated at $x_{l+p}$ and $x_{l}$. Moreover, considering a parameterdependent matrix $P(\zeta)$ in the Lyapunov function (9) instead of a fixed one $P \succ 0 \in \mathbb{S}^{n}$ it is possible to reduce conservativeness.

\subsection{Proposed Conditions}

The proposed condition to design the control gain $\mathcal{K}$ such that the eigenvalues of (6) belong to a given region $\mathscr{D}(\alpha, r)$ in the complex plane is stated in Theorem 1.

Theorem 1. If there exist matrices $P(\zeta) \in \mathbb{S}^{n}, H \in \mathbb{R}^{n \times n}$ and $\mathcal{L} \in \mathbb{R}^{m p \times n}$ such that (11) hold. Then, the control gain $\mathcal{K}=\mathcal{L} H^{-1}$ ensures the closed-loop system $(6)$ is $\mathscr{D}(\alpha, r)$ stable.

$$
\left(\begin{array}{c}
r^{2}\left(-H-H^{\top}+P(\zeta)\right) \\
\mathcal{A}(\zeta) H-\alpha H+\mathcal{B}(\zeta) \mathcal{L}-P(\zeta)
\end{array}\right) \prec 0
$$

Proof. Assume that condition (11) holds. It follows that $H+H^{\top} \succ P(\zeta) \succ 0$, which ensures the regularity of $H$. Thus, from $\mathcal{K}=\mathcal{L} H^{-1}$, (11) can be written as follows:

$$
\left(\begin{array}{c}
r^{2}\left(-H-H^{\top}+P(\zeta)\right) \\
\left(\mathcal{A}(\zeta)+\mathcal{B}(\zeta) \mathcal{K}-\alpha I_{n}\right) H-P(\zeta)
\end{array}\right) \prec 0 .
$$

From the property:

inequality (12) imply

$$
H^{\top} P(\zeta)^{-1} H \succeq H+H^{\top}-P(\zeta),
$$

$$
\left(\begin{array}{c}
-r^{2} H^{\top} P(\zeta)^{-1} H \\
\left(\mathcal{A}(\zeta)+\mathcal{B}(\zeta) \mathcal{K}-\alpha I_{n}\right) H-\stackrel{\bullet}{P}(\zeta)
\end{array}\right) \prec 0 .
$$

Multiplying the last inequality with $\left(\begin{array}{cc}H^{-\top} & 0 \\ 0 & P(\zeta)^{-1}\end{array}\right)$ on the left and its transpose on the right gives

$$
\left(\begin{array}{c}
-r^{2} P(\zeta)^{-1} \\
P(\zeta)^{-1} \mathcal{A}_{c l}(\zeta)-\alpha P(\zeta)^{-1}-P(\zeta)^{-1}
\end{array}\right) \prec 0 .
$$

where $\mathcal{A}_{c l}(\zeta) \triangleq \mathcal{A}(\zeta)+\mathcal{B}(\zeta) \mathcal{K}$.

Let $\lambda$ be any eigenvalue of $\mathcal{A}_{c l}(\zeta)$ and $v \in \mathbb{C}^{n}$ be a nonzero vector such that $v^{H} \mathcal{A}_{c l}(\zeta)^{\top}=\lambda v^{H}$. Multiplying (13) with $I_{2} \otimes v^{H}$ on the left and its transpose on the right gives

$$
v^{H} P(\zeta)^{-1} v\left(\begin{array}{cc}
-r^{2} & \lambda-\alpha \\
\lambda-\alpha & -1
\end{array}\right) \prec 0 .
$$

As $P(\zeta)^{-1} \succ 0$, from Schur complement, it is immediate to conclude that $|\lambda-\alpha|<r$. Thus, since this is true for any eigenvalue of $\mathcal{A}_{c l}(\zeta)$, all of them belong to $\mathscr{D}(\alpha, r)$. This completes the proof.

Remark 1. In Theorem 1 , the control gain $\mathcal{K}$ is computed independently of the parameter-dependent matrix $P(\zeta)$. Performing this computation based on the matrix transformation in terms of $H$ contributes to reduce conservativeness.

Remark 2. If the region is selected as $\mathscr{D}(0,1)$, Theorem 1 corresponds to a standard robust stabilization condition with respect to the Lyapunov function candidate (9). To see that, apply a Schur complement in (13) such that:

$$
\mathcal{A}_{c l}(\zeta)^{\top} P(\zeta)^{-1} \mathcal{A}_{c l}(\zeta)-P(\zeta)^{-1} \prec 0 .
$$

Multiplying the last inequality by $x_{l}^{\top}$ on the left and its transpose on the right and noting that $x_{l+p}^{\top}=x_{l}^{\top} \mathcal{A}_{c l}(\zeta)^{\top}$, one has:

$$
x_{l+p}^{\top} P(\zeta)^{-1} x_{l+p}<x_{l}^{\top} P(\zeta)^{-1} x_{l} .
$$

By adding the term $\sum_{i=1}^{p-1} x_{l+i}^{\top} P(\zeta)^{-1} x_{l+i}$ on both sides of (14), it follows that $\Delta V=V\left(x_{l+1}\right)-V\left(x_{l}\right)<0$, with $V\left(x_{l}\right)$ defined in (9). This proves that the origin of $(6)$ is asymptotically stable.

\subsection{Obtaining LMI Design Conditions}

The condition in Theorem 1 is given in terms of the parameter $\zeta$. By exploiting the convexity property of polytopic uncertainties, a procedure to obtain a set of tractable LMI conditions is presented here. For motivation, consider the following parameter-dependent inequality

$$
\Upsilon(\zeta)=\sum_{\mathbf{i} \in \mathbb{I}_{p}} \zeta_{\mathbf{i}} \Upsilon^{[\mathbf{i}]} \prec 0 .
$$

To solve the above inequality using a finite set of LMIs, it is sufficient to evaluate the negative definiteness of the matrices corresponding to the vertices:

$$
\Upsilon^{\left[\left(i_{p-1}, \ldots, i_{0}\right)\right]} \prec 0
$$

for all $i_{p-1}, \ldots, i_{0} \in I_{[1, N]}$, which corresponds to solve $N^{p}$ LMIs. However, in this case, increasing either the number of polytope vertices $N$ or the system period $p$ implies in exponential growth in the number of LMIs, possibly leading to conservatism. On the other hand, inequality (15) can be equivalently written as follows:

$$
\Upsilon(\zeta)=\sum_{\mathbf{i} \in \mathbb{I}_{p}} \zeta_{\mathbf{i}} \Upsilon^{[\mathbf{i}]}=\sum_{\mathbf{j} \in \mathbb{I}_{p}^{+}} \zeta_{\mathbf{j}} \sum_{\mathbf{k} \in \mathscr{P}(\mathbf{j})} \Upsilon^{[\mathbf{k}]},
$$

where

$$
\mathbb{I}_{p}^{+} \triangleq\left\{\mathbf{i} \in \mathbb{I}_{p}: i_{j} \leq i_{j-1}, j \in I_{[p-1,1]}\right\}
$$

is the set of "upper-triangle" indexes and $\mathscr{P}(\mathbf{i}) \subset \mathbb{I}_{p}$ is the set of permutations among the entries of a given multiindex i (see Sala and Ariño (2007) for details).

Based on the discussion above, the procedure to obtain a finite set of LMI conditions is stated in the following lemma.

Lemma 2. The parameter-dependent inequality (15) is satisfied if the following set of LMIs hold:

$$
\sum_{\mathbf{k} \in \mathscr{P}(\mathbf{j})} \Upsilon^{[\mathbf{k}]} \prec 0,
$$

for all $\mathbf{j} \in \mathbb{I}_{p}^{+}$.

Proof. The proof is direct from (17).

Remark 3. The condition in Lemma 2 can lead to less conservative results than (16) because it does not require all vertices to be negative definite, only appropriate vertices combinations that ensure the negativity of (11). In addition, the number of LMIs to ensure (11) is reduced from $N^{p}$ to $\frac{(N+p-1) !}{p !(N-1) !}$, implying reduction of computational burden.

For instance, a set of LMIs to solve Theorem 1 can be obtained with Lemma 2 defining

$$
\Upsilon(\zeta)=\left(\begin{array}{cc}
r^{2}\left(-H-H^{\top}+P(\zeta)\right) & \bullet \\
\mathcal{A}(\zeta) H-\alpha H+\mathcal{B}(\zeta) \mathcal{L} & -P(\zeta)
\end{array}\right)
$$




\section{ILLUSTRATIVE EXAMPLE}

In this section, a numerical example is presented to illustrate the effectiveness of the proposed conditions. The LMIs were implemented in MATLAB using YALMIP toolbox (Löfberg, 2004) and Mosek solver (MOSEK, 2017).

Consider the following 3-periodic uncertain discrete-time system from Farges et al. (2007):

$$
\begin{aligned}
A_{0}=\left(\begin{array}{cc}
-3-\delta & 2 \\
-3 & 3
\end{array}\right), & B_{0}=\left(\begin{array}{l}
1 \\
\beta
\end{array}\right) \\
A_{1}=\left(\begin{array}{cc}
-1-\delta & 2 \\
0.5 & 0
\end{array}\right), & B_{1}=\left(\begin{array}{c}
1 \\
-\frac{3 \beta+2}{10}
\end{array}\right) \\
A_{2}=\left(\begin{array}{cc}
1-\delta & 2 \\
2.5 & 3
\end{array}\right), & B_{2}=\left(\begin{array}{c}
\frac{\beta+1}{2} \\
1
\end{array}\right)
\end{aligned}
$$

where $\delta$ and $\beta$ are time-invariant parameters satisfying $|\delta| \leq \bar{\delta}$ and $0.2 \leq \beta \leq 0.8$, respectively. The parameter bounds are the same as considered by Ebihara et al. (2015). This system can be easily put in the form of (1) using 4 polytope vertices, $N=4$. For simplicity, the TIR (6) is constructed assuming $\tau=0$. Their vertices are defined by:

$$
\begin{aligned}
& \mathcal{A}^{\left[\left(i_{2}, i_{1}, i_{0}\right)\right]}=A_{2}^{\left[i_{2}\right]} A_{1}^{\left[i_{1}\right]} A_{0}^{\left[i_{0}\right]} \\
& \mathcal{B}^{\left[\left(i_{2}, i_{1}, i_{0}\right)\right]}=\left(A_{2}^{\left[i_{2}\right]} A_{1}^{\left[i_{1}\right]} B_{0}^{\left[i_{0}\right]} A_{2}^{\left[i_{2}\right]} B_{1}^{\left[i_{1}\right]} B_{2}^{\left[i_{2}\right]}\right),
\end{aligned}
$$

for $i_{0}, i_{1}, i_{2} \in I_{[1,2]}$. Recall that $\mathbf{i}=\left(i_{2}, i_{1}, i_{0}\right)$.

Firstly, select the region $\mathscr{D}(0,1)$ in Theorem 1 such that the controller (5) ensure the origin of system (6) is asymptotically stable. LMI conditions are obtained employing Lemma 2. In this case, the maximum uncertainty bound $\bar{\delta}$ for feasibility of Theorem 1 is $\bar{\delta}=0.46$. On the other hand, if a fixed quadratic matrix $P$ was considered, it is obtained $\bar{\delta}=0.37$. This shows the advantage of employing a parameter-dependent Lyapunov function.

To further illustrate the application of Theorem 1, consider $\mathscr{D}(0.2,0.65)$, a similar region as considered in Mao and $\mathrm{Chu}$ (2009). For this region, the maximum bound $\bar{\delta}$ for the uncertainty such that the LMI conditions are feasible is $\bar{\delta}=0.26$. The obtained control gains are the following:

$$
\mathcal{K}=\left(\begin{array}{l}
K_{0} \\
K_{1} \\
K_{2}
\end{array}\right)=\left(\begin{array}{ll}
1.8676 & -1.5197 \\
3.1888 & -4.2712 \\
4.5405 & -4.1778
\end{array}\right)
$$

On the other hand, for a fixed quadratic matrix $P$ in Theorem 1, the maximum uncertainty bound is $\bar{\delta}=0.21$.

To verify the obtained result, the closed-loop eigenvalues for $\bar{\delta}=0.26$ obtained with several values of $\delta$ and $\beta$ generated using a uniform distribution is shown in Figure 2. Clearly, the eigenvalues for all the generated uncertainties are within the specified region $\mathscr{D}(0.2,0.65)$.

The closed-loop state trajectories and control signal for system (1) with matrices defined in (18) are shown in Figure 3 for different uncertainties generated under a uniform distribution. The initial condition for all simulations is $x_{0}=[2,-2]^{\top}$. The gray area represents the one standard deviation around the mean trajectory obtained from 200 simulations with different uncertainties. It illustrates the effectiveness of the sampled state-feedback controller designed using Theorem 1 on stabilizing the uncertain periodic system, while respecting the eigenvalues placement

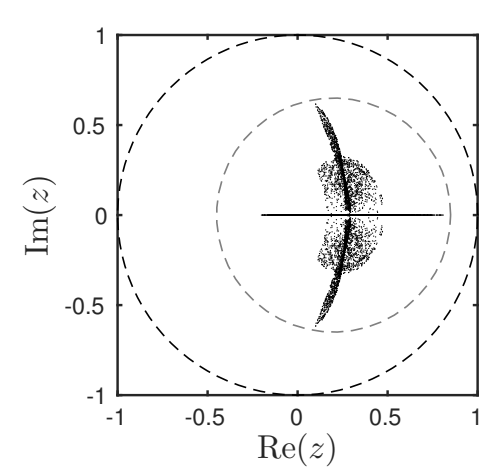

Figure 2. Closed-loop eigenvalues distribution for $|\delta| \leq 0.26$. The gray dashed circle represents the region $\mathscr{D}(0.2,0.65)$.

specifications. The number of performed simulations was selected such that a statistically significant result could be reported based on the sample standard deviation.
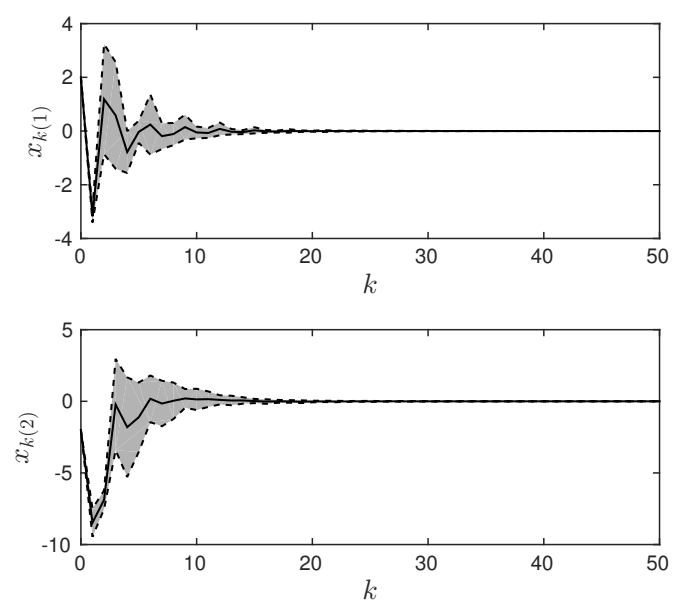

(a) States

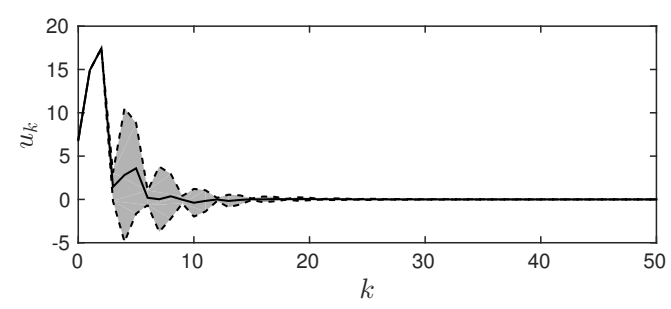

(b) Control signal

Figure 3. Closed-loop trajectories for different values of $\delta$ and $\beta$ within their designed bounds. The mean and standard deviation for all trajectories are shown for each time instant. The region is $\mathscr{D}(0.2,0.65)$.

To highlight the interest in applying the eigenvalue assignment, a comparison is performed with a stabilization strategy. For that, consider $\bar{\delta}=0.26$. After generating 200 values of $\delta$ and $\beta$ within their bounds, the parameter $\theta$ corresponding to the eigenvalue with maximum real part was selected. The parameter for $\mathscr{D}(0,1)$ is $\theta=$ $[0.02,0.01,0.96,0.01]^{\top}$ and the one for $\mathscr{D}(0.2,0.65)$ is $\theta=[0.04,0.93,0.01,0.02]^{\top}$. The closed-loop trajectories for these parameters are shown in Figure 4. The initial condition is $x_{0}=[2,-2]^{\top}$. It is clear that the SFC de- 
signed to allocate the eigenvalues in the region $\mathscr{D}(0.2,0.65)$ can lead to faster stabilization and less oscillation. This shows that performance specifications such as decay rate and damping ratio can be assigned with the proposed eigenvalue assignment approach.

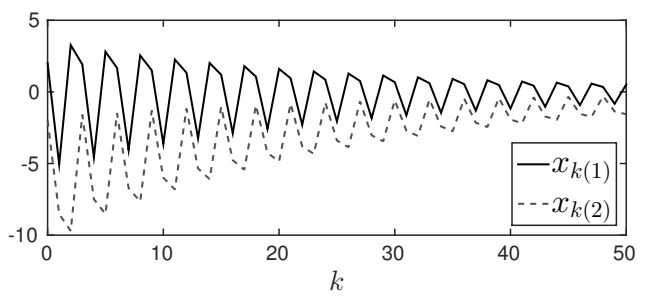

(a) $\mathscr{D}(0,1)$

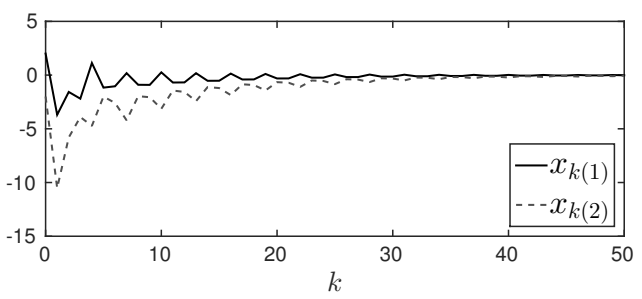

(b) $\mathscr{D}(0.2,0.65)$

Figure 4. Closed-loop trajectories for (a) stabilization and (b) eigenvalue assignment approaches.

\section{CONCLUDING REMARKS}

This paper has addressed the problem of robust eigenvalue assignment via sampled state-feedback control for linear discrete-time periodic systems subject to polytopic uncertainties. A sufficient $\mathscr{D}(\alpha, r)$-stability condition has been proposed for the uncertain time-invariant reformulation obtained from the sampled state-feedback law applied to the linear polytopic discrete-time periodic system. A systematic procedure has been employed to derive LMI design conditions and the proposal effectiveness was demonstrated with numerical simulations. In future works, we aim to extend the proposed conditions to stabilize unstable periodic orbits of nonlinear discrete-time dynamical systems with chaotic sets.

\section{REFERENCES}

Agulhari, C.M. and Lacerda, M.J. (2018). Robust performance for LPV periodic discrete-time systems. In 2018 American Control Conference (ACC), 2029-2034.

Agulhari, C.M. and Lacerda, M.J. (2019). Observer-based state-feedback control design for LPV periodic discretetime systems. European Journal of Control., 49, 1-14.

Bittanti, S. and Colaneri, P. (2009). Periodic Systems: Filtering and Control, volume 5108985. Springer Science \& Business Media.

Bittanti, S. and Cuzzola, F.A. (2002). Periodic active control of vibrations in helicopters: a gain-scheduled multi-objective approach. Control Engineering Practice, 10(10), 1043-1057.

Chagas, T., Bliman, P.A., and Kienitz, K. (2018). Stabilization of periodic orbits of discrete-time dynamical systems using the Prediction-Based Control: New control law and practical aspects. Journal of The Franklin Institute, 355(12), 4771-4793.
Chilali, M. and Gahinet, P. (1996). $H_{\infty}$ design with pole placement constraints: An LMI approach. IEEE Transactions on Automatic Control, 41(3), 358-367.

Colaneri, P. (1991). Output stabilization via pole placement of discrete-time linear periodic systems. IEEE Transactions on Automatic Control, 36(6), 739-742.

De Souza, C.E. and Trofino, A. (2000). An LMI approach to stabilization of linear discrete-time periodic systems. International Journal of Control, 73(8), 696-703.

Ebihara, Y., Peaucelle, D., and Arzelier, D. (2011). Periodically time-varying memory state-feedback controller synthesis for discrete-time linear systems. Automatica, 47(1), 14-25.

Ebihara, Y., Peaucelle, D., and Arzelier, D. (2015). S-Variable Approach to LMI-Based Robust Control. Springer.

Farges, C., Peaucelle, D., Arzelier, D., and Daafouz, J. (2007). Robust H2 performance analysis and synthesis of linear polytopic discrete-time periodic systems via LMIs. Systems \& Control Letters, 56(2), 159-166.

Keles, N.A., Lacerda, M.J., and Agulhari, C.M. (2019). Robust performance and observer based control for periodic discrete-time uncertain systems. Mathematical Problems in Engineering, 2019.

Kruszewski, A., Wang, R., and Guerra, T.M. (2008). Nonquadratic stabilization conditions for a class of uncertain nonlinear discrete time TS fuzzy models: A new approach. IEEE Transactions on Automatic Control, $53(2), 606-611$.

Löfberg, J. (2004). YALMIP: A toolbox for modeling and optimization in MATLAB. In 2004 IEEE International Conference on Robotics and Automation, volume 3, 284289. Taipei, Taiwan.

Lv, L., Duan, G., and Zhou, B. (2010). Parametric pole assignment and robust pole assignment for discrete-time linear periodic systems. SIAM Journal on Control and Optimization, 48(6), 3975-3996.

Mao, W.J. and Chu, J. (2009). $\mathscr{D}$-stability and $\mathscr{D}$ stabilization of linear discrete time-delay systems with polytopic uncertainties. Automatica, 45(3), 842-846.

Meyer, R. and Burrus, C. (1975). A unified analysis of multirate and periodically time-varying digital filters. IEEE Transactions on Circuits and Systems, 22(3), 162168.

MOSEK, A. (2017). The MOSEK optimization toolbox for MATLAB manual. Version 8.1. Http://docs.mosek.com/8.1/toolbox/index.html.

Sala, A. and Ariño, C. (2007). Asymptotically necessary and sufficient conditions for stability and performance in fuzzy control: Applications of Polya's theorem. Fuzzy Sets and Systems, 158(24), 2671-2686.

Sreedhar, J. and Van Dooren, P. (1993). Pole placement via the periodic Schur decomposition. In 1993 American Control Conference (ACC), 1563-1567.

Trégouët, J.F., Arzelier, D., Peaucelle, D., Ebihara, Y., Pittet, C., and Falcoz, A. (2012). Robust $H_{\infty}$ performance of periodic systems with memory: New formulations, analysis and design results. In 2012 IEEE 51st IEEE Conference on Decision and Control (CDC), 7781-7786. IEEE.

Yang, Y. (2018). An efficient LQR design for discrete-time linear periodic system based on a novel lifting method. Automatica, 87, 383-388. 